\title{
Pseudogap in $\mathrm{Fe}_{2}$ VGa: NMR evidence
}

\author{
C. S. Lue* and Joseph H. Ross, Jr. ${ }^{\dagger}$ \\ Department of Physics, Texas A\&M University, College Station, Texas 77843-4242
}

(Received 6 March 2000; revised manuscript received 21 June 2000; published 9 January 2001)

\begin{abstract}
We report the results of a ${ }^{51} \mathrm{~V}$ and ${ }^{69} \mathrm{Ga}$ nuclear magnetic resonance (NMR) study of $\mathrm{Fe}_{2} \mathrm{VGa}$ at temperatures between 4 and $450 \mathrm{~K}$. The presence of magnetic antisite defects is deduced from the NMR linewidth, which displays a Curie-law temperature dependence. The absence of associated NMR shifts indicates the material to be intrinsically nonmagnetic. At low temperatures the NMR spin-lattice relaxation rate exhibits Korringa behavior, indicating a small carrier density at the Fermi level. At elevated temperatures, the Knight shifts and NMR relaxation rates go over to a thermally activated response, a semiconductor-like behavior. These results are consistent with pseudogap features identified by recent band structure calculations. The Fermi level density of states deduced from NMR is considerably smaller than given by the specific heat coefficient, $\gamma$. The electronic properties are compared to the isostructural semimetal $\mathrm{Fe}_{2} \mathrm{VAl}$.
\end{abstract}

DOI: 10.1103/PhysRevB.63.054420

PACS number(s): 75.20.En, 71.20.Lp

\section{INTRODUCTION}

The physics of $d$-electron intermetallics with electronic gaps or pseudogaps near the Fermi level continues to attract attention. Such gaps, in materials containing localized magnetic orbitals, may be due to hybridization or to Kondo-type singlet formation, and there is a continuous range of behavior between these two extremes. ${ }^{1}$ Recently, the Heusler-type $\mathrm{Fe}_{2} \mathrm{VAl}$ compound has been of interest due to its unusual transport and thermodynamic properties, ${ }^{2}$ resembling those of the $3 d$ Kondo insulator FeSi in many ways. ${ }^{3}$ Nuclear magnetic resonance ${ }^{4-6}$ (NMR) and optical conductivity ${ }^{7}$ for $\mathrm{Fe}_{2} \mathrm{VAl}$ have confirmed that this material possess a pseudogap in the Fermi-level density of states (DOS). Similarly, the isostructural compound $\mathrm{Fe}_{2} \mathrm{VGa}$ is predicted from band-structure calculations to be semimetallic, ${ }^{8,9}$ with a small indirect band overlap at the Fermi level. Transport measurements ${ }^{10}$ indicate a possible metal-insulator transition in this material at low temperatures. However, transport measurements are subject to extrinsic effects, due mainly to incomplete ordering of the $\mathrm{Fe}$ and $\mathrm{V}$ sublattices.

NMR is a local probe which can provide intrinsic information, despite the presence of impurities and incomplete ordering. In this investigation, we thus employed NMR techniques to probe the electronic characteristics in $\mathrm{Fe}_{2} \mathrm{VGa}$. Measurements extending from $4.2 \mathrm{~K}$ to $450 \mathrm{~K}$ allowed us to examine the nature of the pseudogap in this material. The results support the assignment of $\mathrm{Fe}_{2} \mathrm{VGa}$ as a band semimetal, with a finite DOS at the Fermi level within a pseudogap.

\section{EXPERIMENTAL DETAILS}

The sample studied here was prepared from $99.97 \% \mathrm{Fe}$, 99.7\% V and $99.9 \%$ Ga by mixing appropriate amounts of elemental metals. They were placed in a water-cooled copper crucible and then were melted several times in an Ar arcmelting furnace. The resulting ingot was annealed in a vacuum-sealed $\left(10^{-5}\right.$ torr) quartz tube at $600^{\circ} \mathrm{C}$ for two days, and then further annealed at $400{ }^{\circ} \mathrm{C}$ for more than $12 \mathrm{~h}$ followed by furnace cooling. The same preparation tech- nique has been used in other studies of this material, ${ }^{11}$ which is known to form in a single-phase L2 ${ }_{1}$ (Heusler-type) structure. The sample was powdered for NMR measurements, with a grain size of $50-100 \mu \mathrm{m}$. Wavelength dispersive spectroscopy showed these grains to be uniform in composition, with an approximate 1 at. \% deficit of Ga. No signs of a second phase were seen in this analysis.

A $\mathrm{Cu} K \alpha$ x-ray analysis on a powdered sample from the annealed ingot was consistent with the expected L2 ${ }_{1}$ structure, with four strong reflections indexed to (220), (400), (422), and (440) planes. The determined lattice parameter, $a=5.77 \AA$, is equal to that reported in the literature, ${ }^{10,12,13}$ with no other phases apparent in the spectrum. X-ray powder intensities calculated by standard means were in very good agreement with the observed intensities. However, calculated intensities were also found to be relatively insensitive to ordering on the $\mathrm{Fe}$ and $\mathrm{V}$ sublattices, due to the nearness $\mathrm{Fe}$ and $\mathrm{V}$ in the Periodic Table. Thus with a single wavelength $\mathrm{x}$-ray measurement the order parameter for $\mathrm{L} 2{ }_{1}$ order is difficult to obtain; ${ }^{13}$ a better measure is provided by the NMR results described below, which indicate the Fe concentration on $\mathrm{V}$ sites to be a few percent, which is also consistent with deductions based on magnetization measurements. ${ }^{10}$

NMR experiments were performed using a 9-T homebuilt pulse NMR spectrometer. ${ }^{51} \mathrm{~V}$ and ${ }^{69} \mathrm{Ga}$ NMR spectra were detected at approximately 101 and $91 \mathrm{MHz}$, respectively, in constant field. The powder mixed with granular quartz was placed in a plastic vial for 4 to $300 \mathrm{~K}$ measurements, while we put the specimen in a Teflon tube for high temperature purposes. Both sample holders showed no observable ${ }^{51} \mathrm{~V}$ and ${ }^{69} \mathrm{Ga}$ NMR signals.

Knight shifts were determined from the position of the maximum of each spectrum with respect to an aqueous $\mathrm{AlCl}_{3}$ solution reference, using 11.093, 10.219, and $11.193 \mathrm{MHz} / \mathrm{T}$ as the gyromagnetic ratios $\left(\gamma_{n} / 2 \pi\right)$ for ${ }^{27} \mathrm{Al},{ }^{69} \mathrm{Ga}$, and ${ }^{51} \mathrm{~V}$, respectively. The spin-lattice relaxation rates $\left(T_{1}\right.$ 's) were measured using the inversion recovery method. We recorded the signal strength by integrating the spin echo FFT of the ${ }^{51} \mathrm{~V}$ and ${ }^{69} \mathrm{Ga}$ lines. For the recovery of the $-1 / 2 \leftrightarrow+1 / 2$ central transition, the $T_{1}$ 's were extracted by fitting to differ- 


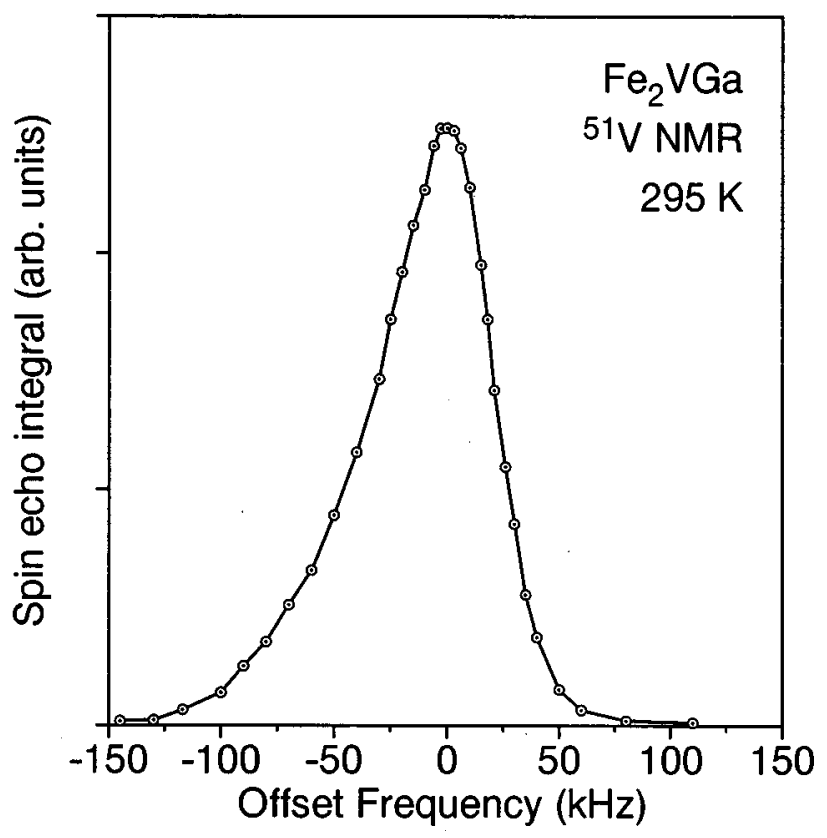

FIG. 1. Room-temperature ${ }^{51} \mathrm{~V}$ NMR powder pattern of $\mathrm{Fe}_{2} \mathrm{VGa}$.

ent multiexponential curves, ${ }^{14}$ as appropriate for $I=7 / 2{ }^{51} \mathrm{~V}$ and $I=3 / 2{ }^{69} \mathrm{Ga}$, with $T_{1}$ as a parameter.

\section{RESULTS}

A ${ }^{51} \mathrm{~V}$ room-temperature powder pattern is shown in Fig. 1, measured from spin-echo integration versus frequency. The ${ }^{69} \mathrm{Ga}$ lineshape exhibits similar behavior. The ordered $\mathrm{L} 2{ }_{1}$ structure contains an equal number of $\mathrm{V}$ and $\mathrm{Ga}$ sites, both with local octahedral symmetry. For this situation, quadrupole splitting vanishes, and a single narrow line is expected for both ${ }^{51} \mathrm{~V}$ and ${ }^{69} \mathrm{Ga}$, as indeed was observed. From the pulse-length dependence of the spin echo, we find that the center of both lines responds as a $-1 / 2 \leftrightarrow+1 / 2$ central transition, while the shoulders have somewhat longer $90^{\circ}$ pulse lengths, characteristic of satellite transitions randomly broadened by defects. ${ }^{15}$ Thus we treated the center of the line as a central transition.

\section{A. NMR linewidths}

We have found the NMR linewidths to be temperaturedependent, with a Curie-type behavior consistent with the presence of local magnetic defects. As a measure of the NMR line shape, we obtained the full width at half maximum (FWHM) of the ${ }^{51} \mathrm{~V}$ NMR line, which is plotted against temperature in Fig. 2. The data can be fit to a temperature-independent width plus a term proportional to $1 / T$, as plotted by the dotted curve in Fig. 2. Such $1 / T$ behavior can be produced by dilute paramagnetic impurities, as previously established. ${ }^{16,17}$ Since the overall shift of the NMR line does not exhibit Curie-type $1 / T$ behavior (as described below), dipole coupling to the paramagnetic impurities is indicated, since dipole broadening has zero spatial

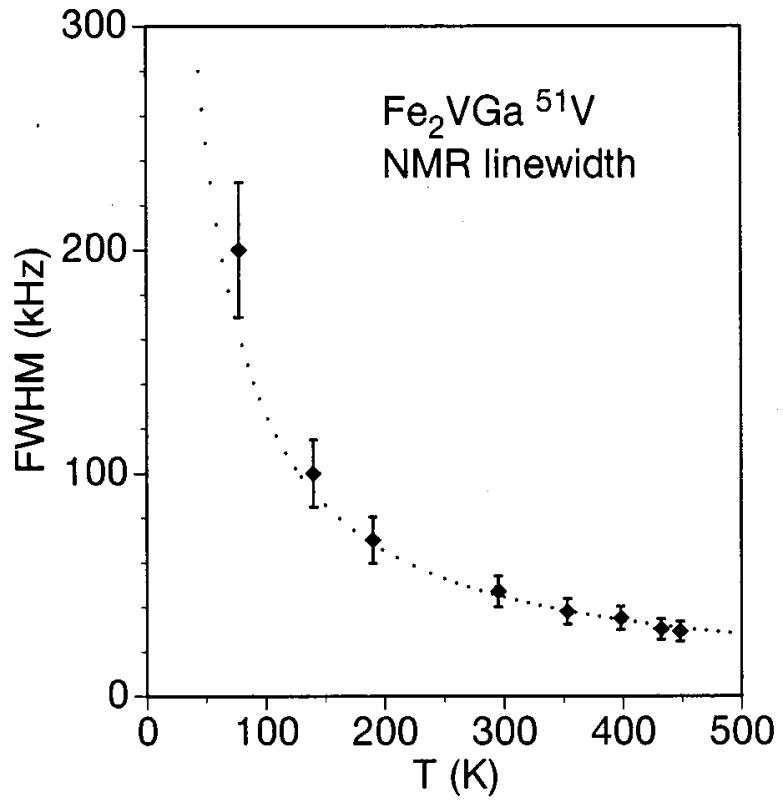

FIG. 2. ${ }^{51} \mathrm{~V}$ FWHM linewidth of $\mathrm{Fe}_{2} \mathrm{VGa}$ vs temperature. Dotted curve: Curie-type behavior described in the text.

average, and hence gives no net shift. Dipole coupling can dominate in low-carrier-density materials, rather than the RKKY interaction which often dominates for impurities in metals.

Electronic structure calculations for $\mathrm{Fe}_{2} \mathrm{VGa}$ indicate the material to be nonmagnetic in the ordered $\mathrm{L}_{2}$ configuration, ${ }^{8,9}$ but with $\mathrm{Fe}$ atoms assuming a magnetic moment when placed on the $\mathrm{V}$ site. A calculated value ${ }^{9}$ for the moment of such wrong-site $\mathrm{Fe}$ atoms is $3.2 \mu_{B}$. Furthermore, a field-dependent specific heat study of isostructural $\mathrm{Fe}_{2} \mathrm{VAl}$ indicated the presence of defects with a moment of $3.7 \mu_{B}$, which were associated with such antisite $\mathrm{Fe}$ atoms. ${ }^{18} \mathrm{We}$ attribute the broadening observed here to this type of defect, and have used the value $3.7 \mu_{B}$ to estimate their concentration.

Broadening by random paramagnetic impurities has been treated rigorously only in the dilute limit, ${ }^{17}$ although Monte Carlo methods have been used for higher concentrations, ${ }^{19}$ demonstrating a change from Lorentzian to Gaussian lineshapes as one goes from dilute to more concentrated situations. We find the central portions of the $\mathrm{Fe}_{2} \mathrm{VGa}$ lines to be close to Gaussian in shape, and therefore outside of the dilute limit, in contrast to results obtained for $\mathrm{Fe}_{2} \mathrm{VAl}$, which shows Lorentzian lineshapes for both ${ }^{51} \mathrm{~V}$ and ${ }^{27} \mathrm{Al} .{ }^{4,5} \mathrm{As}$ an approximate measure, however, we have taken the dipole broadening function as calculated in the dilute limit by Walstedt and Walker, ${ }^{17}$ and compared to the FWHM observed here. The result, which is the dotted curve in Fig. 2, gives an impurity concentration $f=4 \%$ per formula unit. This result can be compared to $f=0.4 \%$ found by specific heat methods in $\mathrm{Fe}_{2} \mathrm{VAl}{ }^{18}$ The temperature-independent portion of the linewidth obtained in this fit is $3 \mathrm{kHz}$, corresponding to a relatively small contribution due to sample inhomogeneities other than the magnetic defects. 


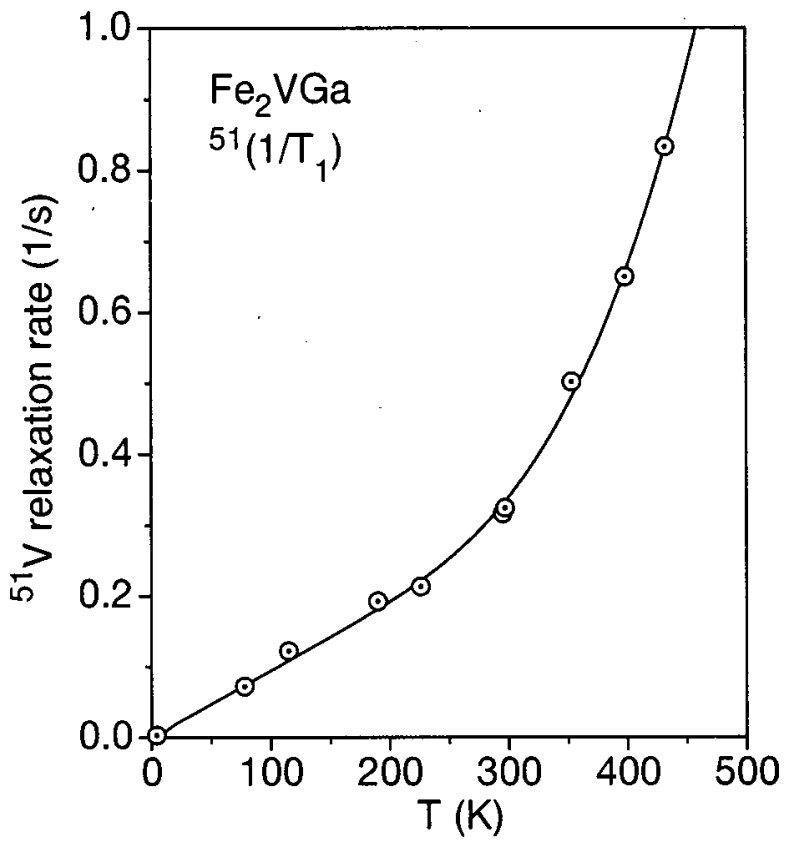

FIG. 3. Temperature-dependent ${ }^{51} \mathrm{~V}$ spin-lattice relaxation rates for $\mathrm{Fe}_{2} \mathrm{VGa}$. Solid curve: fit to the Korringa plus activated behavior.

Although the dilute-limit broadening function gives only an approximate value in the present case, the concentration of these defects is clearly larger in $\mathrm{Fe}_{2} \mathrm{VGa}$ than $\mathrm{Fe}_{2} \mathrm{VAl}$, consistent with the deviation from Lorentzian shape observed here. Endo et al. ${ }^{10}$ assumed a moment equal to $5 \mu_{B}$ per antisite defect, and obtained a concentration of $2.6 \%$ per formula unit from magnetization measurements in similarly prepared $\mathrm{Fe}_{2} \mathrm{VGa}$, quite consistent with the present result. From magnetization and specific heat studies of these systems, we have further concluded that superparamagnetic clusters of these defects are also present, ${ }^{20}$ having considerably larger effective moments, but these are quite dilute and do not affect the NMR results described here.

\section{B. Spin-lattice relaxation rates}

Figure 3 shows the ${ }^{51} \mathrm{~V}$ spin-lattice relaxation rates, denoted ${ }^{51}\left(1 / T_{1}\right)$, measured as a function of temperature. At low temperatures, ${ }^{51}\left(1 / T_{1}\right)$ is proportional to the temperature, a characteristic signature of the Korringa relaxation process due to metallic electrons. From a straight-line fit to the data below $250 \mathrm{~K}$, we obtained ${ }^{51}\left(1 / T_{1 K} T\right)=9.4$ $\times 10^{-4} \mathrm{~s}^{-1} \mathrm{~K}^{-1}$, where the subscript $K$ denotes the Korringa process. Normally, the spin contribution to $1 / T_{1 K} T$ is proportional to the Fermi-level DOS. Comparison to the experimental value for $\mathrm{V}$ metal, ${ }^{21} 1 / T_{1} T=1.27 \mathrm{~s}^{-1} \mathrm{~K}^{-1}$, shows the $\mathrm{Fe}_{2} \mathrm{VGa}$ value to be small, consistent with electronic structure calculations ${ }^{8,9}$ indicating a small band overlap at the Fermi level in this material.

$T_{1}$ measurements for ${ }^{69} \mathrm{Ga}$ (not shown) produced results similar to those for ${ }^{51} \mathrm{~V}: 1 / T_{1}$ linear in $T$ at low temperatures, and increasing much more rapidly at higher temperatures. A fit to the low-temperature data yielded ${ }^{69}\left(1 / T_{1 K} T\right)=6.5$ $\times 10^{-3} \mathrm{~s}^{-1} \mathrm{~K}^{-1}$, about 7 times larger than ${ }^{51}\left(1 / T_{1 K} T\right)$.
However, the hyperfine coupling for Ga-s orbitals is approximately 70 times larger than the V- $d$ core polarization term, ${ }^{22}$ so the corresponding Fermi-surface weight in Ga-s orbitals is considerably smaller than in $\mathrm{V}-d$ orbitals. This is also consistent with calculations, ${ }^{8,9}$ which have indicated the carriers to be in small pockets dominated by $\mathrm{Fe}$ and $\mathrm{V} d$ orbits.

An earlier report ${ }^{6}$ showed a larger, non-Korringa-like ${ }^{51}\left(1 / T_{1}\right)$ in $\mathrm{Fe}_{2} \mathrm{VGa}$ at low temperatures. A local moment contribution due to defects was inferred, ${ }^{6}$ but such a contribution to our data is apparently smaller than the Korringa term, because of sample and/or magnetic field dependence. Note that in a previous study ${ }^{4}$ we found the ${ }^{27} \mathrm{Al}\left(1 / T_{1}\right)$ in $\mathrm{Fe}_{2} \mathrm{VAl}$ to be smaller than ${ }^{51}\left(1 / T_{1}\right)$, with a non-Korringa temperature dependence indicating ${ }^{27}\left(1 / T_{1}\right)$ to be dominated by local moments, while ${ }^{51}\left(1 / T_{1}\right)$ is dominated by Korringa relaxation.

Relaxation due to paramagnetic defects is caused by fluctuating moments, often producing a Debye peak in the nuclear $T_{1}^{-1}$ due to the slowing down of fluctuations. ${ }^{23}$ This explains the peak in ${ }^{27}\left(1 / T_{1}\right)$ for $\mathrm{Fe}_{2} \mathrm{VAl}^{4}$ Values of $T_{1}$ due to this mechanism will be nearly equivalent for different nuclei with similar gyromagnetic ratios. ${ }^{23}$ By contrast, in $\mathrm{Fe}_{2} \mathrm{VGa}$ we have different rates for ${ }^{51} \mathrm{~V}$ and ${ }^{69} \mathrm{Ga}$, and both are linear in $T$ at low temperatures, the hallmark of a Korringa process. The light nucleus ${ }^{27} \mathrm{Al}$ has a smaller hyperfine coupling to conduction electrons, allowing paramagnetic relaxation to be relatively more important. Because $1 / T_{1}$ terms are additive, any unresolved paramagnetic contribution to ${ }^{51}\left(1 / T_{1}\right)$ and ${ }^{69}\left(1 / T_{1}\right)$ will in principle cause an overestimation of the Korringa term, and the resulting DOS. However, for the reasons described above, it is clear that the Korringa process is the most important for both ${ }^{51} \mathrm{~V}$ and ${ }^{69} \mathrm{Ga}$.

Above $250 \mathrm{~K},{ }^{51}\left(1 / T_{1}\right)$ rises rapidly, with an activated temperature dependence. This is the characteristic behavior for semiconductors, ${ }^{24}$ with the increase in relaxation rate due to an increase in the number of carriers because of thermal excitation across an energy gap. It is easy to reconcile the metallic behavior observed at low temperatures with the high-temperature semiconducting behavior by assuming a two-band model, with one band overlapping the Fermi level while the second band is separated from the Fermi level by an energy gap, $\Delta \epsilon$. In this case, the relaxation rate is given by $^{24}$

$$
\frac{1}{T_{1} T}=\frac{1}{T_{1 K} T}+C_{1} T e^{-\Delta \epsilon / k_{B} T}
$$

where $1 / T_{1 K} T$ is the Korringa value obtained above, and the second term is due to the band edge separated from the Fermi level. This form assumes an effective-mass approximation for the band edge, with $C_{1}$ a constant depending upon the effective mass, as well as other factors. We fixed the ${ }^{51} T_{1 K}$ value obtained from the low-temperature fit, and found that Eq. (1) gives good agreement with the data, shown as the solid curve in Fig. 3. The result of the leastsquares fit is $\Delta \epsilon=0.11 \mathrm{eV}$. 


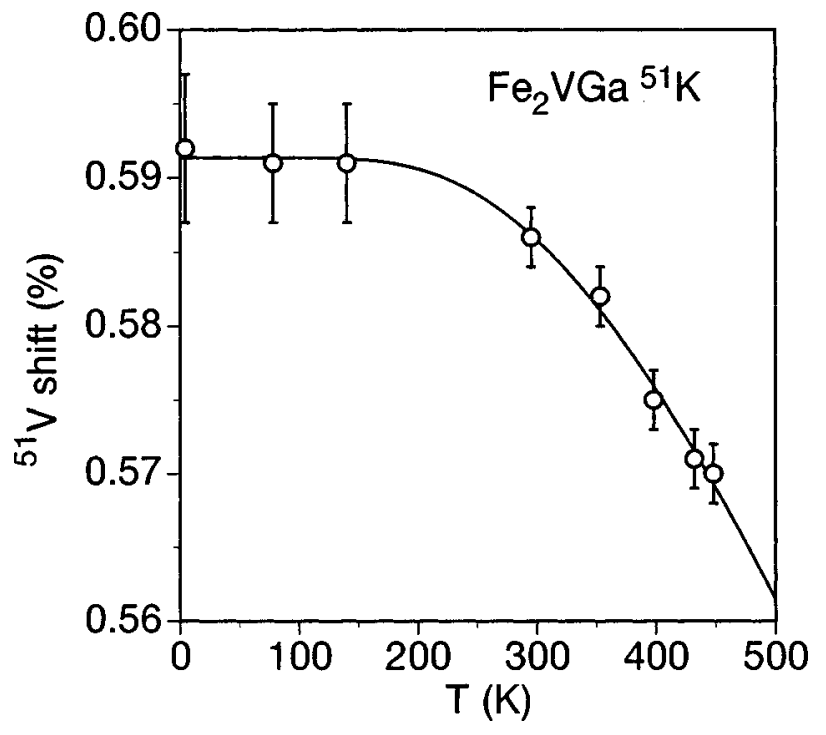

FIG. 4. Temperature dependence of ${ }^{51} \mathrm{~V}$ Knight shifts of $\mathrm{Fe}_{2}$ VGa. Solid curve: fit to Eq. (2).

\section{Knight shifts}

The temperature dependence of the ${ }^{51} \mathrm{~V}$ Knight shift (denoted ${ }^{51} K$ ) is displayed in Fig. 4. The characteristics of this behavior are a temperature-independent shift observed at low temperatures, and a shift to lower frequencies with increasing temperature above $250 \mathrm{~K}$, which can be fit to an activated curve. The ${ }^{69} \mathrm{Ga}$ Knight shift $\left({ }^{69} \mathrm{~K}\right)$ exhibits a similar trend, not shown here.

Below $250 \mathrm{~K},{ }^{51} \mathrm{~K}$ and ${ }^{69} \mathrm{~K}$ are $0.59 \%$ and $0.07 \%$, respectively. The temperature-independence of these shifts is characteristic of nonmagnetic materials, and indicates that despite the paramagnetic local-moment defects identified through the linewidths, the bulk of the material is nonmagnetic. Using the measured values of $T_{1 K}$, the Korringa relation $^{22}$ can be used to estimate the expected temperatureindependent metallic contributions to these shifts, $0.005 \%$ and $0.013 \%$ for ${ }^{51} \mathrm{~V}$ and ${ }^{69} \mathrm{Ga}$, respectively. While electronelectron interactions can enhance such shifts, the shifts observed here can be attributed largely to an orbital mechanism. The orbital shift is a paramagnetic chemical shift due to orbitals away from the band edges, and is not associated with conducting states. For comparison, ${ }^{69} \mathrm{Ga}$ chemical shifts in III-V zinc-blende semiconductors range up to $0.03 \%,{ }^{25}$ while a ${ }^{51} \mathrm{~V}$ orbital shift of approximately $0.63 \%$ in A15structure $\mathrm{V}_{3} \mathrm{Ga}$ is apparent from NMR in the superconducting state, where the spin susceptibility is zero. ${ }^{26}$ Thus the large value for ${ }^{51} K$ does not signify strongly metallic behavior.

Above $250 \mathrm{~K},{ }^{51} \mathrm{~K}$ shifts to lower frequencies with increasing temperature. A negative ${ }^{51} \mathrm{~V}$ shift reflects an increase in the spin susceptibility, due to the negative V-hyperfine coupling for the core-polarization mechanism. ${ }^{22}$ Thus, the decrease in ${ }^{51} K$ can be attributed to a thermally activated increase in the density of carriers, also responsible for the increase in $T_{1}$. The Knight shift in this case can be written $^{24}$

$$
K=K_{o}+C_{2} \sqrt{T} e^{-\Delta \epsilon / k_{B} T},
$$

where $K_{o}$ is a combination of orbital and temperatureindependent metallic shifts, $C_{2}$ is a negative constant, and $\Delta \epsilon$ is the excitation energy as defined before. The solid curve in Fig. 4 represents a fit to Eq. (2), yielding $\Delta \epsilon$ $=0.095 \mathrm{eV}$, consistent with the result obtained via spinlattice relaxation.

\section{DISCUSSION}

Magnetic properties of $\mathrm{Fe}_{3-x} \mathrm{~V}_{x} \mathrm{Ga}$ and $\mathrm{Fe}_{3-x} \mathrm{~V}_{x} \mathrm{Al}$ are quite similar, except that $\mathrm{Fe}_{3-x} \mathrm{~V}_{x} \mathrm{Ga}$ maintains a larger moment in the nominally nonmagnetic composition $x=1$, and a Curie temperature of $15 \mathrm{~K}$ was deduced from features observed in the bulk magnetization of this composition. ${ }^{10}$ The nature of this transition is particularly interesting in light of the apparent metal-insulator transition and large giant magnetoresistance observed near $\mathrm{T}_{c} \cdot{ }^{10,27}$ However, from the $\mathrm{ab}$ sence of additional shifts or splitting of the low-temperature NMR lines, ordered $\mathrm{Fe}_{2} \mathrm{VGa}$ appears nonmagnetic. The transition must be a property of the defects, and not, for instance, a bulk transition induced by the presence of defects. A calculation for ordered $\mathrm{Fe}_{2} \mathrm{VGa}$ indicated a residual moment of $0.03 \mu_{B}$ per Fe compensated by $-0.06 \mu_{B}$ on $\mathrm{V},{ }^{9}$ but frozen moments of this size will broaden or shift the ${ }^{51} \mathrm{~V}$ NMR resonance by about $5.8 \mathrm{MHz}$, given the hyperfine coupling ${ }^{28}$ $H_{h f}^{d}=-86 \mathrm{kG}$. Such shifts are not observed here, indicating ordered $\mathrm{Fe}_{2} \mathrm{VGa}$ to be nonmagnetic.

A further question is whether $\mathrm{Fe}_{2} \mathrm{VGa}$ is nonmagnetic due to hybridization effects, or another mechanism such as Kondo lattice behavior. ${ }^{1}$ Indeed, the reported value of $\gamma$, the $T$-linear specific heat coefficient, is $20 \mathrm{~mJ} / \mathrm{mol} \mathrm{K}^{2}$, corresponding to an electronic mass enhancement of order 100 over calculated values. ${ }^{27}$ The Korringa contribution to ${ }^{51}\left(1 / T_{1}\right)$ can provide a measure of $g\left(\epsilon_{f}\right)$, as reported previously for $\mathrm{Fe}_{2} \mathrm{VAl}^{4,5}$ For $d$-spin relaxation in metals, the ${ }^{51} \mathrm{~V}$ term is ${ }^{22}$

$$
\left(T_{1 K} T\right)^{-1}=2 h k_{B}\left[\gamma_{n} H_{h f}^{d} g_{V}\left(\epsilon_{f}\right)\right]^{2} q,
$$

where $g_{V}\left(\epsilon_{f}\right)$ is the $\mathrm{V}$ partial $d$-spin contribution, $q$ is a factor equal to the reciprocal of the degeneracy, ${ }^{21}$ and $H_{h f}^{d}$ is the core polarization hyperfine field. $H_{h f}^{d}=-86 \mathrm{kG}$, calculated for $\mathrm{V}$ metal, ${ }^{28}$ provides a good estimate for this parameter. Furthermore, band-structure calculations ${ }^{8,9}$ indicate an $e_{g}$ orbital symmetry for electron pockets which would dominate the ${ }^{51} \mathrm{~V}$ relaxation. These pockets have degeneracy of 2 , giving $q=1 / 2$. Using these parameters we obtain $g_{V}\left(\epsilon_{f}\right)$ $=0.085$ states/eV atom, which can be compared to $g_{V}\left(\epsilon_{f}\right)$ $=0.023$ states/eV atom measured this way in $\mathrm{Fe}_{2} \mathrm{VAl}^{5}{ }^{5} \mathrm{Weht}$ and Pickett ${ }^{8}$ calculated $g\left(\epsilon_{f}\right)=0.1$ total states/eV atom for $\mathrm{Fe}_{2} \mathrm{VAl}$, and have indicated that $\mathrm{Fe}_{2} \mathrm{VGa}$ possesses a larger band overlap at $\epsilon_{f}$. The measured values are thus in reasonable agreement.

While Eq. (3) assumes the electrons to be noninteracting, electron-electron interactions can lead to an enhancement of $1 / T_{1 K} T$. This enhancement can take a number of forms; in spin fluctuation theory ${ }^{29}$ the enhancement is proportional to 
the enhancement of the magnetic susceptibility or to its square root, and the enhancement of $1 / T_{1 K} T$ would be expected to be significant in cases where the enhancement of $\gamma$ is significant. For cases in which the relaxation is dominated by local moments, the Korringa relation no longer holds. The ${ }^{51}\left(1 / T_{1 K} T\right)$ observed for $\mathrm{Fe}_{2} \mathrm{VGa}$ is in reasonable agreement with the results of band theory with no enhancement term, indicating hybridization to play a dominant role in this case.

Away from $\epsilon_{f}$, a conduction-band edge having little dispersion in reciprocal space between the $\mathrm{X}$ and $\Gamma$ points is calculated to lie approximately $0.1 \mathrm{eV}$ above $\epsilon_{f}$ in $\mathrm{Fe}_{2} \mathrm{VGa}$ (measured from Fig. 11 in Ref. 9). The separation of this band edge from $\epsilon_{f}$ is in very satisfactory agreement with the values, $\Delta \epsilon=0.11 \mathrm{eV}$ and $0.095 \mathrm{eV}$, obtained from the activated behavior of $T_{1}$ and the Knight shift, respectively. Therefore, thermal excitation of carriers to this band at high temperatures provides a good explanation for the behavior of both $T_{1}$ and the Knight shift.

The measured values for $\Delta \epsilon$ are not equal, with the activation energy obtained from $T_{1}$ slightly exceeding the value obtained from the Knight shift. A larger difference appears in one-dimensional spin-gap systems, where the $T_{1}$ activation energy exceeds the gap obtained from Knight shifts by a factor of approximately 1.5 , compared to a ratio very close to 1 for most materials. ${ }^{30}$ This may result from the separation of singlet and triplet excitations in these materials, among other possibilities. ${ }^{31}$ An explanation for the ratio observed here, 1.15 , would be a tendency towards similar spin excitations in $\mathrm{Fe}_{2} \mathrm{VGa}$, and hence the possible influence of Kondo-type interactions. ( $\mathrm{In} \mathrm{Fe}_{2} \mathrm{VAl}$ the ratio is 1.3 , measured independently both by ${ }^{51} \mathrm{~V}$ and ${ }^{27} \mathrm{Al} \mathrm{NMR} .{ }^{4}$ ) Another possible explanation is nonparabolicity of the band edge, modifying the energy dependence of the DOS, giving correction terms in Eqs. (1) and (2). ${ }^{24}$ Further experiments to address this issue

*Present address: Department of Physics, National Sun-Yat-Sen University, Kaohsiung, Taiwan. Electronic address: chinshan@rossgroup.tamu.edu

†Electronic address: jhross@tamu.edu

${ }^{1}$ A.C. Hewson, The Kondo Problem to Heavy Fermions (Cambridge University Press, Cambridge, England, 1993); L. Degiorgi, Rev. Mod. Phys. 71, 687 (1999).

${ }^{2}$ Y. Nishino, M. Kato, S. Asano, K. Soda, M. Hayasaki, and U. Mizutani, Phys. Rev. Lett. 79, 1909 (1997).

${ }^{3}$ Z. Schlesinger, Z. Fisk, Hai-Tao Zhang, M.P. Maple, J.F. DiTusa, and G. Aeppli, Phys. Rev. Lett. 71, 1748 (1993).

${ }^{4}$ C.-S. Lue and Joseph H. Ross, Jr., Phys. Rev. B 58, 9763 (1998).

${ }^{5}$ C.S. Lue and Joseph H. Ross, Jr., Phys. Rev. B 61, 9863 (2000).

${ }^{6}$ Kiyoshi Ooiwa and Keizo Endo, J. Magn. Magn. Mater. 177-181, 1443 (1998).

${ }^{7}$ H. Okamura, J. Kawahara, T. Nanba, S. Kimura, K. Soda, U. Mizutani, Y. Nishino, M. Kato, I. Shimoyama, H. Miura, K. Fukui, K. Nakagawa, H. Nakagawa, and T. Kinoshita, Phys. Rev. Lett. 84, 3674 (2000).

${ }^{8}$ Ruben Weht and W.E. Pickett, Phys. Rev. B 58, 6855 (1998).

${ }^{9}$ A. Bansil, S. Kaprzyk, P.E. Mijnarends, and J. Tobola, Phys. Rev. B 60, 13396 (1999).

${ }^{10}$ K. Endo, H. Matsuda, K. Ooiwa, M. Iijima, K. Ito, T. Goto, and would be most interesting. In either case, the measurements do not depart significantly from expectations based on band theory, again indicating hybridization to be the dominant interaction. Thus we characterize $\mathrm{Fe}_{2} \mathrm{VGa}$ as a hybridizationgap semimetal.

There remains an inconsistency between the small $g\left(\epsilon_{f}\right)$ deduced from NMR and the large observed $\gamma$. The reported value, $20 \mathrm{~mJ} / \mathrm{mol} \mathrm{K} \mathrm{K}^{2}$ corresponds to an electronic mass enhancement of order $100,{ }^{27}$ compared to the value from NMR relaxation rates. However, it is likely that a considerable portion of the observed $\gamma$ results from extrinsic effects, leading to a Schottky-type enhancement, and false heavy Fermion behavior, as found in $\mathrm{Fe}_{2} \mathrm{VAl} .{ }^{18}$ To provide further information, a magnetic study focusing on magnetic defects and clusters is underway.

\section{CONCLUSIONS}

NMR measurements have provided a local picture of the electronic properties of $\mathrm{Fe}_{2} \mathrm{VGa}$, indicating that despite the presence of magnetic antisite defects, $\mathrm{Fe}_{2} \mathrm{VGa}$ is essentially nonmagnetic, with properties quite similar to those calculated via band-structure methods. Both the Knight shifts and spin lattice relaxation rates are understood in terms of semimetallic characteristics, with a small band overlap at $\boldsymbol{\epsilon}_{f}$ falling within a pseudogap formed by nearby bands. No large enhancement of the Korringa relaxation process was found, indicating the pseudogap to be due to hybridization, rather than Kondo, effects.

\section{ACKNOWLEDGMENTS}

This work was supported in part by the Robert A. Welch Foundation (A-0514).

${ }^{11}$ Nobuo Kawamiya, Yoichi Nishino, Mitsuyasu Matsuo, and Shigeru Asano, Phys. Rev. B 44, 12406 (1991).

${ }^{12}$ K.H.J. Buschow and P.G. van Engen, J. Magn. Magn. Mater. 25, 90 (1981).

${ }^{13}$ P.J. Webster and K.R.A. Ziebeck, Phys. Lett. 98A, 51 (1983); P.J. Webster, Contemp. Phys. 10, 559 (1969).

${ }^{14}$ A. Narath, Phys. Rev. 162, 320 (1967).

${ }^{15} \mathrm{O}$. Kanert and M. Mehring, in NMR: Basic Principles and Progress, edited by E. Fluck and R. Kosfeld (Springer-Verlag, New York, 1971), Vol. 3, p. 1.

${ }^{16}$ C. Kittel and Elihu Abrahams, Phys. Rev. 90, 238 (1953).

${ }^{17}$ R.E. Walstedt and L.R. Walker, Phys. Rev. B 9, 9732 (1974).

${ }^{18}$ C.S. Lue, Joseph H. Ross, Jr., C.F. Chang, and H.D. Yang, Phys. Rev. B 60, R13941 (1999).

${ }^{19}$ D.R. Noakes, Phys. Rev. B 44, 5064 (1991).

${ }^{20}$ C.S. Lue, Joseph H. Ross, Jr., K.D.D. Rathnayaka, D.G. Naugle, S.Y. Wu, and W.-H. Li, J. Phys.: Condens. Matter (to be published).

${ }^{21}$ Y. Yafet and V. Jaccarino, Phys. Rev. A 133, A1630 (1964).

${ }^{22}$ Metallic Shifts in NMR, edited by G.C. Carter, L.H. Bennett, and D.J. Kahan (Pergamon, Oxford, 1977).

${ }^{23}$ A. Abragam and M. Goldman, Nuclear Magnetism: Order and Disorder (Clarendon, Oxford, 1982); I.P. Goudemond, J.M. 
Keartland, M.J.R. Hoch, and G.A. Saunders, Phys. Rev. B 56, R8463 (1997).

${ }^{24}$ N. Bloembergen, Physica (Amsterdam) 20, 1130 (1954); Dieter Wolf, Spin Temperature and Nuclear-spin Relaxation in Matter (Clarendon, Oxford, 1979).

${ }^{25}$ Oc Hee Han, Hye Kyung C. Timken, and Eric Oldfield, J. Chem. Phys. 89, 6046 (1988).

${ }^{26}$ A.C. Clogston, A.C. Gossard, V. Jaccarino, and Y. Yafet, Phys. Rev. Lett. 9, 262 (1962).

${ }^{27}$ K. Endo, H. Matsuda, K. Ooiwa, M. Iijima, T. Goto, K. Sato, and
I. Umehara, J. Magn. Magn. Mater. 177-181, 1437 (1998).

${ }^{28}$ H. Ebert, H. Winter, and J. Voitländer, J. Phys. F: Met. Phys. 16, 1133 (1986).

${ }^{29}$ Toru Moriya, Spin Fluctuations in Itinerant Electron Magnetism (Springer-Verlag, Berlin, 1985).

${ }^{30}$ Subir Sachdev and Kedar Demle, Phys. Rev. Lett. 78, 943 (1997); Y. Itoh and H. Yasuoka, J. Phys. Soc. Jpn. 66, 334 (1997).

${ }^{31}$ K. Magishi, S. Matsumoto, Y. Kitaoka, K. Ishida, K. Asayama, M. Uehara, T. Nagata, and J. Akimitsu, Phys. Rev. B 57, 11533 (1998). 